\title{
Prediction of Hottest Spot Temperature (HST) in Power and Station Transformers
}

\author{
M. K. Pradhan and T. S. Ramu
}

\begin{abstract}
The degradation of electrical insulation in transformers is more often due to thermal stress. In this paper, authors have attempted to work out a method of assessing the magnitude of temperature and the hottest region in the body of a large transformer with a reasonable degree of accuracy. A theoretical model has been developed based on boundary value problem of heat conduction in transformer winding using finite integral transform techniques. The model requires, in addition to electrical parameters of the transformer, information on the actual design data. The authors believe that this model predicts HST with precision and also indicates a possibility of online data acquisition.
\end{abstract}

Index Terms-Finite transforms, H S location, heat transfer, IEEE loading guide, oil impregnated paper, prediction accuracy.

\section{NOMENCLATURE}

$\alpha_{d} \quad$ Diffusivity of insulation-conductor system $\mathrm{m}^{2} / \mathrm{s}$.

$\alpha_{m}$

$\beta$

Roots of transcendental equation (14).

Coefficient of volumetric expansion of oil.

$\beta_{n} \quad$ Roots of transcendental equation (13).

$\Delta \theta_{b a, r} \quad$ Ultimate bottom oil rise over ambient at rated load ${ }^{\circ} \mathrm{C}$.

$\Delta \theta_{b a} \quad$ Ultimate bottom oil rise over ambient ${ }^{\circ} C$.

$\delta \quad$ Characteristic dimension, used in the computation of $G r_{\delta}, G r_{* \delta}, R a_{\delta}, R a_{* \delta}, N u_{\delta}$, etc. $\delta$ can be disc thickness, height of winding, $(b-a) / 2$ [half of diff. between outer and inner radii of an annular surface or diameter as used in (36) and (37)].

$\ell \quad$ Thickness of the disc or height of layer winding $\mathrm{m}$.

$\gamma \quad$ Temperature rise exponent to bottom oil.

$\mu / \nu \quad$ Dynamic/kinetic viscosity of transformer oil.

$\overline{\bar{F}} \quad$ Combine Fourier and Hankel transform of Initial function $\mathbf{F}$.

$\overline{\overline{\mathbf{g}}}_{0} \quad$ Combine Fourier and Hankel transform of heat source function $\mathbf{g}_{0}$.

$\rho_{\text {oil }} \quad$ Density of transformer oil $\mathrm{kg} / \mathrm{m}^{3}$.

$\tau \quad$ Time constant of solid part of insulation min

$a, b \quad$ Inner and outer radius of the annular disc or layer.

c. $f(\xi) \quad$ Correction factor to $N u_{\delta}$ for cylindrical curvature.

$C_{p c u} \quad$ Specific heat of conductor $J / \mathrm{kg}^{\circ} \mathrm{C}$.

$C_{p e q} \quad$ Equivalent specific heat of conductor and insulation composite system $\mathrm{J} / \mathrm{kg}^{\circ} \mathrm{C}$.

$C_{\text {pin }} \quad$ Specific heat of insulation $J / \mathrm{kg}^{\circ} \mathrm{C}$.

$f_{r} \quad \mathbf{G} r_{D} / \mathbf{R} e^{2}$ relative index of free to forced convection.

Manuscript received September 6, 2002.

The authors are with the Department of High Voltage Engineering, Indian Institute of Science, Bangalore 560012, India (e-mail: manoj@hve.iisc.ernet.in).

Digital Object Identifier 10.1109/TPWRD.2003.817739

$f_{h s t}$
$G_{z}$
$G r_{* \delta}$
$G r_{\delta}$
$k_{i}, h_{i}$,
$\mathbf{f}_{i}$

$K_{c u}$,
$K_{i n}$
$\operatorname{loc}^{n}(r / z)$
$m_{s i}$

$n, m$

$N u_{m}$

$N u_{\delta}$

$q_{w}$

$r_{i}$ 's

$R a_{* \delta}$

$R a_{\delta}$

$T_{a}$

$T_{b}$

$T_{\text {top }}$

$T_{m}$

$t_{c u}, t_{i n}$

$T_{h s}$

$V_{\text {oil }}$

$\mathbf{f}_{i(t: 0)}$

$\mathbf{f}_{i(t: \infty-0)}$

$\mathbf{K}_{0}\left(\beta_{n}, r\right)$

$\mathbf{M}\left(\boldsymbol{\alpha}_{m}, z\right)$

$\mathrm{g}$

hvD

L

$\operatorname{lv} 2 \mathrm{~L}$
Correction factor to resistance change at H S location.

Graetz number.

Grassof number based on constant heat flux. Grassof number based on temperature difference. Thermal conductivity, heat transfer coefficient, and boundary function at different surfaces of annular cylinder, $i=1,2 \rightarrow$ inner, outer cylinder surfaces, $i=3,4 \rightarrow$ bottom, top surfaces.

Thermal conductivity of copper, insulation, $W / m^{\circ} C$.

In Table II, reads as radial position/axial position. Oil temperature gradient along winding height, ${ }^{\circ} \mathrm{C} / \mathrm{m}$.

Index to roots of transcendental (13) and (14).

Mean Nusselt number:

(i) $c . f(\xi) \times 1.5 \times 0.6 \mathbf{R} a_{* \boldsymbol{\delta}}^{0.2}$, for inner and outer cylindrical surfaces of a disc or a layer.

(ii) $0.61 \mathbf{R} a_{* \boldsymbol{\delta}}^{0.2}$, top annular surface of a disc/a layer.

(iii) $0.35 \mathbf{R} a_{* \delta}^{0.2}$, bottom annular surface of a disc/a layer.

(iv) Equation (37) combined free and forced convection (OF).

Local Nusselt number with characteristic dimension $\delta$.

Heat flux in $w m^{-2}$.

$i=1,2 \ldots$, Radii of insulation and conductor layers.

Rayleigh number based on constant heat flux.

Rayleigh number based on temperature difference. Ambient temperature ${ }^{\circ} \mathrm{C}$.

Temperature at the bottom and top of the winding.

$=\left(T_{\text {oil }}+T_{w s}\right) / 2,{ }^{\circ} \mathrm{C},\left(T_{\text {oil }}\right.$, bulk oil temperature $T_{w s}$, winding surface temperature.

Thickness of conductor, insulation.

Hottest spot temperature (HST) ${ }^{\circ} \mathrm{C}$.

Oil velocity inside the cooling duct $\mathrm{m} / \mathrm{s}$.

Temperature profile in the z-direction at $t=0$.

Difference of temperature profile in the z-direction at $t=\infty$ and $t=0$.

Kernel function for finite Hankel transform (FHT).

Kernel function for finite Fourier transform (FFT).

Acceleration due to gravity $\mathrm{m}^{2} / \mathrm{s}$.

High voltage disc winding.

Per unit load.

Second layer of low voltage winding. 
$\mathrm{p} \quad p=225$, for aluminum, $p=234.5$ for copper.

$\mathrm{R} \quad$ Loss ratio = load loss/no load loss.

Re, $P r \quad$ Reynold number, Prandtl number.

\section{INTRODUCTION}

$\mathbf{P}$ OWER and station transformers are expensive and difficult-to-replace elements of a power system. The failure of such equipment would therefore cause long power supply interruptions with consequent loss of revenue, entailing costly repairs besides loss of reliability of power system. In order to detect an impending failure of a power transformer and to avoid catastrophic failures, carefully designed diagnostic testing and condition monitoring (DTCM) practices have been in vogue for some time now. The aim of DTCM is to assess the status of electrical insulation in transformers and other equipment at any given point of time and suggest prognostic measures. The mineral oil impregnated cellulose, (oil impregnated paper, OIP) in its various forms, is the insulation of choice in transformers and more often, this system is given to rapid degradation and inadvertent failure due to thermal effects (temperature rise). Of the many parameters used as reliable indices of the temporal status of insulation, monitoring the temperature of the hottest spot (HST) in the transformer winding is deemed to give a reasonably precise indication of the rapidity of the degradation process.

The current ANSI/IEEE loading guide [1] for mineral oil immersed transformer suggests means of predicting top oil temperature (TOT) by a physical model recently proposed by research group at MIT,USA [2]. Further, to reduce the error in such predictions, a neural network approach has been suggested by Daniel [3] and Qing et al. [4]. A perusal of the literature in the field indicates that there is no known procedure for computing HST directly. However, The IEEE loading guide and certain other works [1], [5], [6] give empirical methods for this evaluation. Some time ago it was argued that installation of temperature sensors in the body of insulation could solve this problem. But difficulties were experienced in locating the sensors, and ensuring their reliability.

Opportunity has therefore been taken of the current situation by the authors to suggest alternatives. Authors proposal involves formulation and solution of the heat conduction equations (HCE), considering most of the complexities of the transformer insulation structure and to arrive at the temperature distribution, as well as magnitude of HST. Also proposed is a means of locating the HST in the transformer winding. To handle the complex and composite structure of insulation and conductor, it is necessary to make some simplifying assumptions with minimal loss of generality. In this paper, the steady state and transient thermal performance of transformers under different loading, ambient, and cooling conditions have also been considered in some detail. Section II deals with mathematical formulation of boundary value problem (BVP) and its solution using finite Hankel and Fourier transforms [7] for both steady and unsteady state. Results and discussion of the proposed work have been provided in Section III.

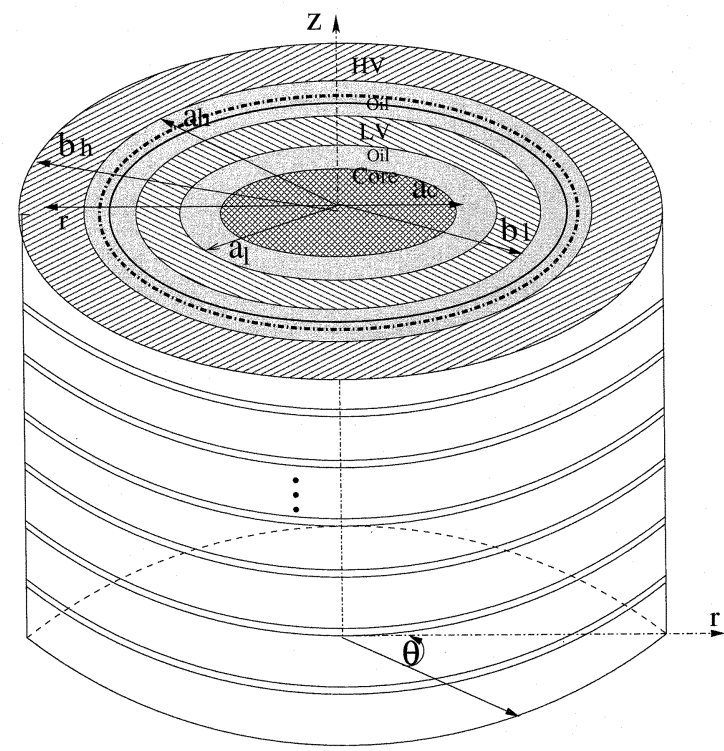

Fig. 1. Simple model of transformer winding, $H V=$ disc, $L V=$ layer.

\section{MATHEMATICAL FORMULATION OF BVP FOR HCE}

The structure of a transformer winding is complex and does not conform to any known geometry in the strict sense. Under fairly general conditions, the transformer windings can be assumed cylindrical in formation; hence, a layer or a disc winding is a finite annular cylinder. The thermal and physical properties of the system would be equivalent to a composite system of insulation and conductor. It has been assumed that heat is generated throughout the body at a constant rate, and oil in the vertical and horizontal ducts take away the heat through the process of convection. However, in an actual transformer winding, the conductor is the only heat source. A simple sketch of model transformer winding is given in Fig. 1. Later in this section, formulations are given for calculating different thermal and physical properties of the system. It has been assumed that temperature is independent of space variable $\theta$, due to the fact that winding structure is symmetrical. The temperature at any point on the periphery of circle for a specific value of $r$ and $z$ is deemed a constant (i.e., presence of spacers has been ignored, thus reducing a three-dimensional (3-D) problem to a two-dimensional (2-D) one with $\mathrm{r}$ and $\mathrm{z}$ as space variables. Dielectric loss in insulation is assumed to be small compared to copper losses in the conductor. Later, it will be shown that this simplification does not influence the final results. The surface of disc or layer has been assumed smooth. Such an assumption gives a pessimistic result, since roughness disturbs viscous sublayer, accounting for a higher heat transfer coefficient. The model in its present form is for individual discsp; however, it can simply be extended to a complete winding after small modifications.

The generalized system of nonhomogeneous HCE with nonhomogeneous boundary condition, in cylindrical coordinate system is, written thus

$$
\frac{\partial}{r \partial r}\left(r \frac{\partial \mathbf{T}}{\partial r}\right)+\frac{\partial^{2} \mathbf{T}}{\partial z^{2}}+\frac{\mathbf{g}_{\mathbf{0}}}{\mathbf{K}}=\frac{1}{\alpha_{d}} \frac{\partial \mathbf{T}}{\partial t}
$$

in the region $a \leq r \leq b, 0 \leq z \leq \ell, t>0$. 
At the inner cylindrical surface $(r=a, t>0)$

$$
-k_{1} \frac{\partial \mathbf{T}}{\partial r}+h_{1} \mathbf{T}=\mathbf{f}_{1}(z, t)
$$

At the outer cylindrical surface $(r=b, t>0)$

$$
k_{2} \frac{\partial \mathbf{T}}{\partial r}+h_{2} \mathbf{T}=\mathbf{f}_{2}(z, t) \text {. }
$$

At the bottom flat surface $(z=0, t>0)$

$$
-k_{3} \frac{\partial \mathbf{T}}{\partial r}+h_{3} \mathbf{T}=\mathbf{f}_{3}(r, t)
$$

At the top flat surface $(z=\ell, t>0)$

$$
k_{4} \frac{\partial \mathbf{T}}{\partial z}+h_{4} \mathbf{T}=\mathbf{f}_{4}(r, t) .
$$

In the region $a \leq r \leq b, 0 \leq z \leq \ell$ and $t=0$

$$
\mathbf{T}=\mathbf{F}(r, z) \text {. }
$$

Equations (1) through (6), represent the general BVP of HCE with convection at all four boundary surfaces. In the above equations, temperature $\mathbf{T}$ is a function of space variables $\mathrm{r}, \mathrm{z}$ and time variable $\mathrm{t}$ (i.e., $\mathbf{T} \equiv \mathbf{T}(r, z, t)$ ). The term $\mathrm{g}_{0}$ is the heat source function assumed constant throughout the volume of a particular disc or layer and $\mathbf{F}(r, z)$ represents the initial function to transient problem of heat conduction. Boundary functions $\mathbf{f}_{1}(z, t)$ and $\mathbf{f}_{2}(z, t)$, derived from Newton's law of cooling are of the form (7), and its degenerate form in steady state are of the form of (8)

$$
\begin{aligned}
\mathbf{f}_{i}(z, t) & \equiv \mathbf{f}_{i(t: 0)}(z)+\mathbf{f}_{i(t: \infty-0)}(z)\left[1-e^{-\frac{t}{\tau}}\right] \\
\mathbf{f}_{i}(z) & =h_{i} \mathbf{T} z_{i}=h_{i} \times\left(T_{b}+m_{s_{i}} z\right)
\end{aligned}
$$

where $\mathbf{f}_{i(t: 0)}(z)$ is the temperature profile at time $(t=0)$ and $\mathbf{f}_{i(t: \infty-0)}(z)$ is the difference of temperature profile at time $(t=$ $\infty)$ and $(t=0)$. The term $T_{b}$ is the temperature at the bottom of the disc or layer, as applicable. Term $m_{s_{i}}$ is the temperature gradient along the winding height (for layer) or along disc thickness for a disc. $\tau$ is the time constant (TC) of the thermal process.

Similarly, functions $\mathbf{f}_{3}$ and $\mathbf{f}_{4}$, representing temperature profiles across bottom and top surfaces, having the form same as shown in (7), with argument " $\mathrm{z}$ " replaced by "r." The corresponding degenerated form in steady state will be as shown in (9), where temperature gradient term $m_{s_{i}}$ has been taken as zero (has been assumed to be constants throughout the surface at a given instant of time)

$$
\mathbf{f}_{j}(r)=h_{j} \times\left(T_{b} \text { or } T_{t o p}\right) .
$$

In general, thermal conductivities (tc) $k_{1} \ldots k_{4}$ are shown different, and are indicated accordingly. But in an actual case, the thermal conductivities in radial directions $\left(k_{1}\right.$ and $\left.k_{2}\right)$ are equal and conductivities in z-direction will also be the same $\left(k_{3}=k_{4}\right)$. Term $\mathbf{K}$ represents resultant thermal conductivity of insulation and conductor system. Heat transfer coefficients (htc) $h_{1} \ldots h_{4}$, are different across all the four surfaces, the values of which can be calculated by using heat transfer empirical relations, given later in this section.

\section{A. Steady State Condition}

If the load is constant over a longer period of time compared to the thermal time constant of the solid part of transformer in- sulation, and if there is no, or very little, variation in temperature of the oil surrounding the winding, the thermal state is called a steady state. While the fluctuation in atmospheric temperature does cause a variation in TOT, the HST is not influenced by a dynamic ambient. However, the effect of cooling fans, if any, is to be considered both in TOT and HST calculations. Using the steady state HCE, the HST can be computed, as under. Degenerate form of general equation (1), in steady state condition is

$$
\frac{\partial}{r \partial r}\left(r \frac{\partial \mathbf{T}}{\partial r}\right)+\frac{\partial^{2} \mathbf{T}}{\partial z^{2}}+\frac{\mathbf{g}_{0}}{\mathbf{K}}=0 ; \quad a \leq r \leq b, 0 \leq z \leq \ell
$$

where the time, $t$ is absent. The applicable boundary conditions are the same as in (2)-(5) except that the time (variable) has been suppressed. These equations can be solved using finite Hankel and Fourier transforms w.r.t the $\mathrm{r}$ and $\mathrm{z}$ variable, respectively. The finite Fourier and Hankel transformation and inverse transformation pairs are given in Appendix A. Finite Hankel and Fourier transforms of steady state (10) have been taken, transformed equation is the form of

$$
\begin{aligned}
\overline{\overline{\mathbf{T}}}\left(\beta_{n}, \alpha_{m}\right)= & \frac{1}{\left(\alpha_{m}^{2}+\beta_{n}^{2}\right)} \\
& \times\left[\left.\frac{\overline{\mathbf{f}}_{1}\left(\alpha_{m}\right)}{k_{1}}\left\{r \mathbf{K}_{0}\left(\beta_{n}, r\right)\right\}\right|_{r=a}\right. \\
& +\left.\frac{\overline{\mathbf{f}}_{2}\left(\alpha_{m}\right)}{k_{2}}\left\{r \mathbf{K}_{0}\left(\beta_{n}, r\right)\right\}\right|_{r=b} \\
& +\left.\frac{\overline{\mathbf{f}}_{3}\left(\beta_{n}\right)}{k_{3}}\left\{\mathbf{M}\left(\alpha_{m}, z\right)\right\}\right|_{z=0} \\
& \left.+\left.\frac{\overline{\mathbf{f}}_{4}\left(\beta_{n}\right)}{k_{4}}\left\{\mathbf{M}\left(\alpha_{m}, z\right)\right\}\right|_{z=\ell}+\frac{\overline{\mathbf{g}}_{0}}{\mathbf{K}}\right]
\end{aligned}
$$

The inversion has been operated on (11). The steady state temperature has been obtained, a double summation series

$$
\mathbf{T}(r, z)=\sum_{n=1}^{\infty} \sum_{m=1}^{\infty} \overline{\overline{\mathbf{T}}}\left(\beta_{n}, \alpha_{m}\right) \mathbf{K}_{0}\left(\beta_{n}, r\right) \mathbf{M}\left(\alpha_{m}, z\right)
$$

where $\mathbf{K}_{0}\left(\boldsymbol{\beta}_{n}, r\right)$ and $\mathbf{M}\left(\alpha_{m}, z\right)$ are kernel function, has been used for finite Hankel and Fourier transform, respectively. In the (11), $\overline{\mathbf{f}}_{1}$ and $\overline{\mathbf{f}}_{2}$ represent the finite Fourier transforms of $\mathbf{f}_{1}$ and $\mathbf{f}_{2} \cdot \overline{\mathbf{f}}_{3}$ and $\overline{\mathbf{f}}_{4}$ are finite Hankel transforms of $\mathbf{f}_{3}$ and $\mathbf{f}_{4} \cdot \overline{\mathbf{g}}_{0}$ is the double transform of source function $\mathbf{g}_{0}$, w.r.t. $\mathbf{K}_{0}\left(\boldsymbol{\beta}_{n}, r\right)$ and $\mathbf{M}\left(\alpha_{m}, z\right)$. To determine the accuracy of estimation, transformation and inverse transformation of different types of functions (boundary, source, and initial function) w.r.t. kernel functions described are considered. Later it has been checked, as to what the number of terms should be, to obtain a desired accuracy. It has been found that numbers $n$ and $m$ between 6 to 8 , give sufficient accuracy. Mathematical expression of the kernel functions described has been included in Appendix A. The parameters $\beta_{n}$ and $\alpha_{m}$, known as the Eigenvalues and are positive roots of transcendental equations (13) and (14), respectively

$$
\begin{aligned}
& \frac{\beta_{n} k_{1} J_{1}\left(\boldsymbol{\beta}_{n} a\right)+h_{1} J_{0}\left(\boldsymbol{\beta}_{n} a\right)}{h_{2} J_{0}\left(\boldsymbol{\beta}_{n} b\right)-k_{2} \boldsymbol{\beta}_{n} J_{1}\left(\boldsymbol{\beta}_{n} b\right)}- \frac{\beta_{n} k_{1} Y_{1}\left(\boldsymbol{\beta}_{n} a\right)+h_{1} Y_{0}\left(\boldsymbol{\beta}_{n} a\right)}{h_{2} Y_{0}\left(\boldsymbol{\beta}_{n} b\right)-k_{2} \boldsymbol{\beta}_{n} Y_{1}\left(\boldsymbol{\beta}_{n} b\right)}=0 \\
& \tan \left(\alpha_{m} \ell\right)-\frac{\alpha_{m}\left(\frac{h_{3}}{k_{3}}+\frac{h_{4}}{k_{4}}\right)}{\alpha_{m}^{2}-\frac{h_{3}}{k_{3}} \frac{h_{4}}{k_{4}}}=0 .
\end{aligned}
$$




\section{B. Unsteady State Condition}

The conduction under unsteady state can also be described by the system of (1) through (6). Time dependent boundary functions were assumed in the form of (7). To solve the unsteady equation, temperature at $t=0$, called initial function, can be calculated by solving the steady state problem of initial state. A polynomial of $\mathrm{r}$ and $\mathrm{z}$ has been found out which approximately represent the temperature profile at $t=0$. It has been checked that by taking only $2_{n d}$ degree polynomial of $\mathrm{z}$ and $6_{t h}$ degree of $r$ is sufficient to provide accuracy up to $99.6 \%$. The solution procedure of the unsteady state problem is exactly similar to steady state case. By applying combine transform (see Appendix A) to general HCE equation (1), transformed equations will be as shown below

$$
\frac{d \overline{\mathbf{T}}\left(\beta_{n}, \alpha_{m}, t\right)}{d t}+\alpha_{d}\left(\alpha_{m}^{2}+\beta_{n}^{2}\right) \overline{\overline{\mathbf{T}}}=\overline{\overline{\mathbf{Q}}}\left(\beta_{n}, \alpha_{m}, t\right)
$$

and $\overline{\overline{\mathbf{T}}}\left(\beta_{n}, \alpha_{m}, t\right)=\overline{\overline{\mathbf{F}}}\left(\beta_{n}, \alpha_{m}\right)$ for $t=0$. where

$$
\begin{aligned}
& \overline{\overline{\mathbf{Q}}}\left(\beta_{n}, \alpha_{m}, t\right)=\left[\left.\frac{\overline{\mathbf{f}}_{1}\left(\alpha_{m}, t\right)}{k_{1}}\left\{r \mathbf{K}_{0}\left(\beta_{n}, r\right)\right\}\right|_{r=a}\right. \\
&+\left.\frac{\overline{\mathbf{f}}_{2}\left(\alpha_{m}, t\right)}{k_{2}}\left\{r \mathbf{K}_{0}\left(\beta_{n}, r\right)\right\}\right|_{r=b} \\
&+\left.\frac{\overline{\mathbf{f}}_{3}\left(\beta_{n}, t\right)}{k_{3}} \mathbf{M}\left(\alpha_{m}, z\right)\right|_{z=0} \\
&\left.+\left.\frac{\overline{\mathbf{f}}_{4}\left(\beta_{n}, t\right)}{k_{4}} \mathbf{M}\left(\alpha_{m}, z\right)\right|_{z=l}+\frac{\overline{\mathbf{g}}_{0}}{\mathbf{K}}\right] \alpha_{d} .
\end{aligned}
$$

The above (15), is a Leibnitz equation, can be solved and equated to give

$$
\begin{aligned}
& \overline{\overline{\mathbf{T}}}\left(\beta_{n}, \alpha_{m}, t\right)=e^{-\alpha_{d}\left(\alpha_{m}^{2}+\beta_{n}^{2}\right) t} \\
& \quad \times\left[\overline{\overline{\mathbf{F}}}\left(\beta_{n}, \alpha_{m}\right)+\int_{0}^{t} \overline{\overline{\mathbf{Q}}}\left(\beta_{n}, \alpha_{m}, t^{\prime}\right) e^{\alpha_{d}\left(\alpha_{m}^{2}+\beta_{n}^{2}\right) t^{\prime}} d t^{\prime}\right] .
\end{aligned}
$$

The temperature distribution $\mathbf{T}(r, z, t)$ is calculated as follows:

$$
\begin{aligned}
& \mathbf{T}(r, z, t)=\sum_{n=1}^{\infty} \sum_{m=1}^{\infty} \mathbf{K}_{0}\left(\beta_{n}, r\right) \mathbf{M}\left(\alpha_{m}, z\right) e^{-\alpha_{d}\left(\alpha_{m}^{2}+\beta_{n}^{2}\right) t} \\
& \quad \times\left[\overline{\overline{\mathbf{F}}}\left(\beta_{n}, \alpha_{m}\right)+\int_{0}^{t} \overline{\overline{\mathbf{Q}}}\left(\beta_{n}, \alpha_{m}, t^{\prime}\right) e^{\alpha_{d}\left(\alpha_{m}^{2}+\beta_{n}^{2}\right) t^{\prime}} d t^{\prime}\right] .
\end{aligned}
$$

\section{Calculation of Heat Transfer Coefficient}

To determine boundary functions $\mathbf{f}_{1}$ to $\mathbf{f}_{4}$, it is necessary to calculate heat transfer coefficients $\left(h_{1}\right.$ to $\left.h_{4}\right)$ across the four surfaces. Difficulty has been encountered in calculation heat transfer coefficient. It is reported elsewhere [8], That it depends on as many as 13 factors (e.g., winding size, type, duct dimensions, oil velocity, type of oil circulation, heat flux distribution, oil thermal properties, etc.). In this work, corrections have been given for temperature dependence of the thermal and physical properties of oil, such as viscosity $(\mu)$, specific heat $\left(C_{p}\right)$, volumetric expansion, and thermal conductivity $\left(k_{o i l}\right)$. It was found that there is negligible effect of specific heat, coefficient of volumetric expansion $(\beta)$, and conductivity in the present working range of loading $\left(110-160^{\circ} \mathrm{C}\right)$. Following are some of
TABLE I

INFLUENCE OF THE VALUES OF H'S AND K'S ON HST

\begin{tabular}{c|c|c|c}
\hline $\mathrm{h}(\%)$ & $\mathrm{k}(\%)$ & $\mathrm{HST}(\%)$ & $\begin{array}{c}\text { Error } \\
\text { Error }\end{array}$ \\
Error & $\begin{array}{c}\text { Error } \\
\text { in }\end{array}$ \\
\hline \hline-10 & 0 & +1.0 & $h_{1}$ only \\
0 & -10 & +0.3 & $k_{1}, k_{2}$ \\
-10 & -10 & +1.3 & $h_{1}, k_{1}, k_{2}$ \\
\hline
\end{tabular}

the heat transfer empirical relations and relevant formulae in natural cooling $(\mathrm{ON})$ mode. These formulae have been used to calculate the htc [9], [10].

i) Local Nusselt number for laminar flow over vertical plates has been shown below

$$
N u_{\delta}=0.6 \mathbf{R} a_{* \delta}^{0.2} \text { or } .6\left(\mathbf{G} r_{* \delta} P_{r}\right)^{0.2}
$$

where $\mathbf{R} a_{* \delta}$ and $\mathbf{G} r_{* \delta}$ are the local Raleigh and Grassof number based on heat flux $\left(q_{w}\right)$ at characteristic dimension $(\delta) . P_{r}$ is the Prandtl number of transformer oil. Expression of Raleigh number based on constant heat flux is

$$
\mathbf{R} a_{* \delta}=\frac{g \beta C_{p} \rho_{o i l}^{2} q_{w} \delta^{4}}{k_{o i l}^{2} \mu}
$$

ii) Mean Nusselt number in this case can be computed as

$$
N u_{m}=1.25\left[N u_{\delta}\right]_{\delta=\ell} .
$$

However, correction to above empirical formula (18), has to be given for cylindrical curvature (r). The correction factor in this case is of the following form:

$$
\text { c.f }(\xi)=\frac{N u_{\delta c y l}}{N u_{\delta v p}}, \quad \text { where } \xi=\frac{2 \sqrt{2}}{G r_{\delta}^{.25}} \times \frac{\delta}{r} .
$$

Here $G r_{\delta}$ is Grassof num. based on temperature difference. Typically, c. $f(\xi) \approx 1+0.12 \xi$, in the range of Prandtl number $\operatorname{Pr}=30$ to 50 . Heat transfer coefficient can be computed as $h_{\delta}=N u_{\delta} K_{o i l} / \delta$, where $h_{\delta}$ represents local coefficient. Mean coefficient $\left(h_{m}\right)$ can be calculated from mean Nusselt number, as in (20). After knowing $h_{m}$ for a particular surface, temperature difference between the winding surface and oil can be found out, dividing the $h_{m}$ by the heat flux through the surface. The heat transfer empirical formulae for calculating htc across top and bottom surface, oil temperature gradient, and viscosity computation at different oil temperature have been provided in the Appendix B. It has been verified that the value of HST is not so sensitive to small error in the calculation of htc. Table I, shows expected Prediction error for different degree of error in the calculation of htc and tc.

\section{RESUlTS AND DiscusSIONS}

\section{A. Steady State Thermal Performance}

The steady state performance has been studied by a software program using Mathematica code. The result obtained seems to be in good agreement with some of the experimental results viz. [5]. The model has been validated on two transformers of ratings 25/32 MVA, (ONAN/ONAF) and 22.5 MVA (ONAN). Authors 


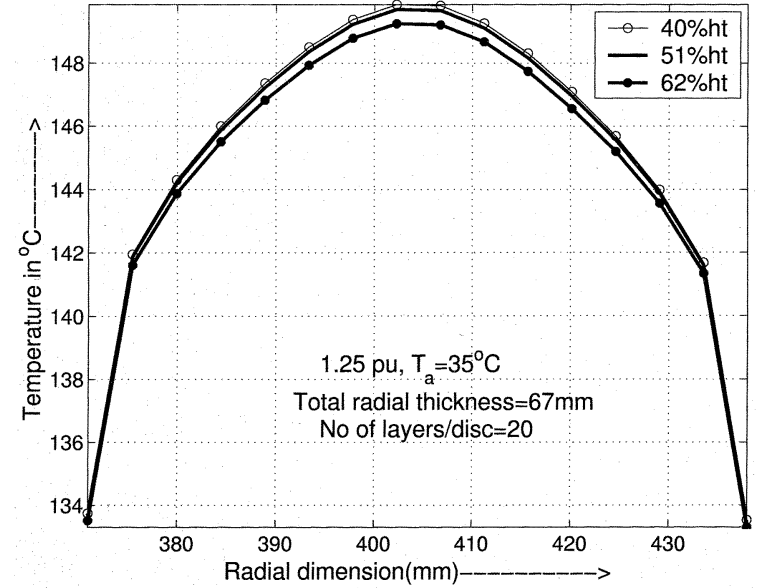

Fig. 2. Radial temperature distribution, hvD, ONAF.

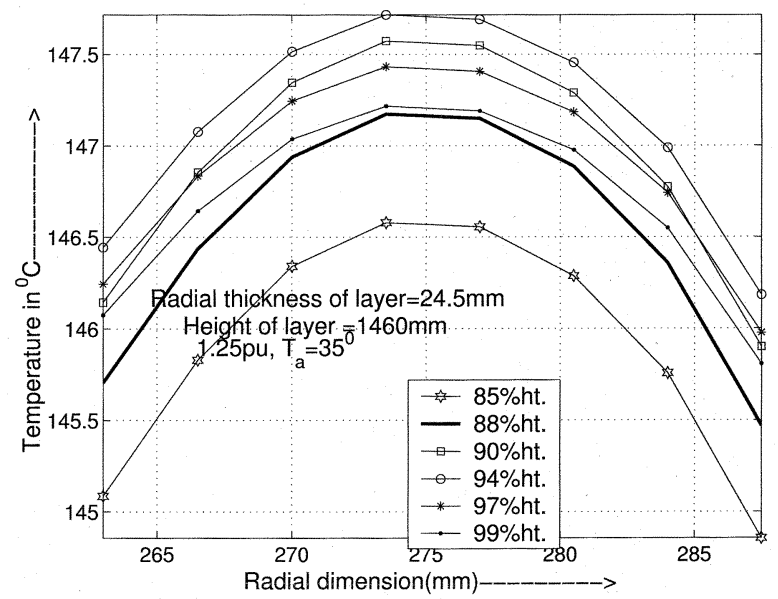

Fig. 3. Radial temperature distribution, lv1 L, ONAF.

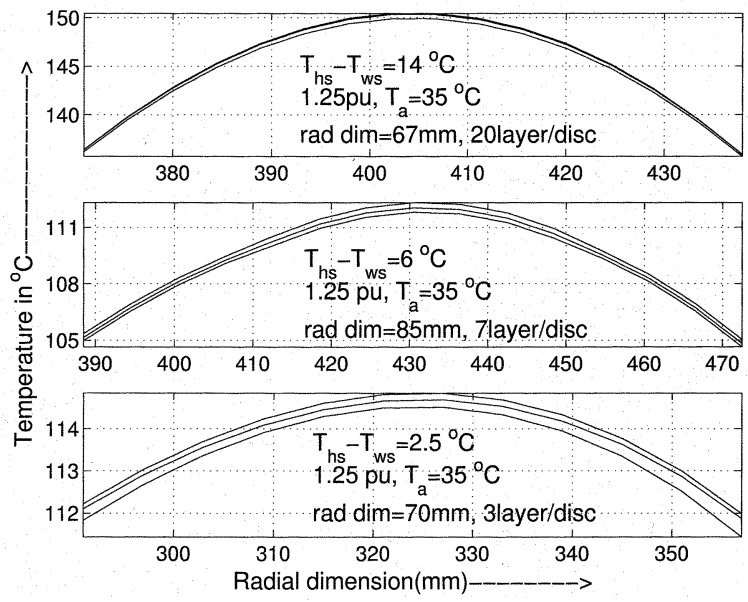

Fig. 4. HST rise above winding surface temperature.

of the present paper are of the view that the methods of estimation of HST suggested here are quite general and are applicable to any oil immersed transformer. The result of radial temperature distribution for a disc and a layer, from a layer winding has been shown in Figs. 2 and 3. The HST rise over winding surface

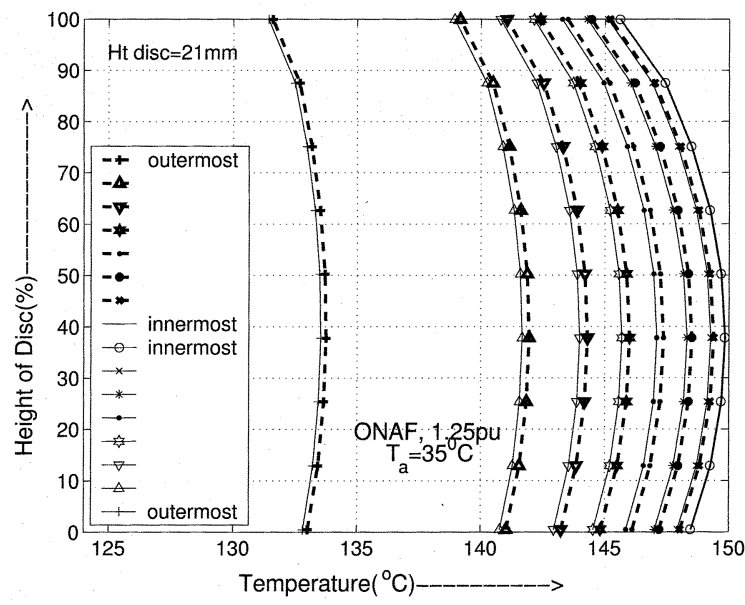

Fig. 5. Axial temperature distribution of an hvD, ONAF.

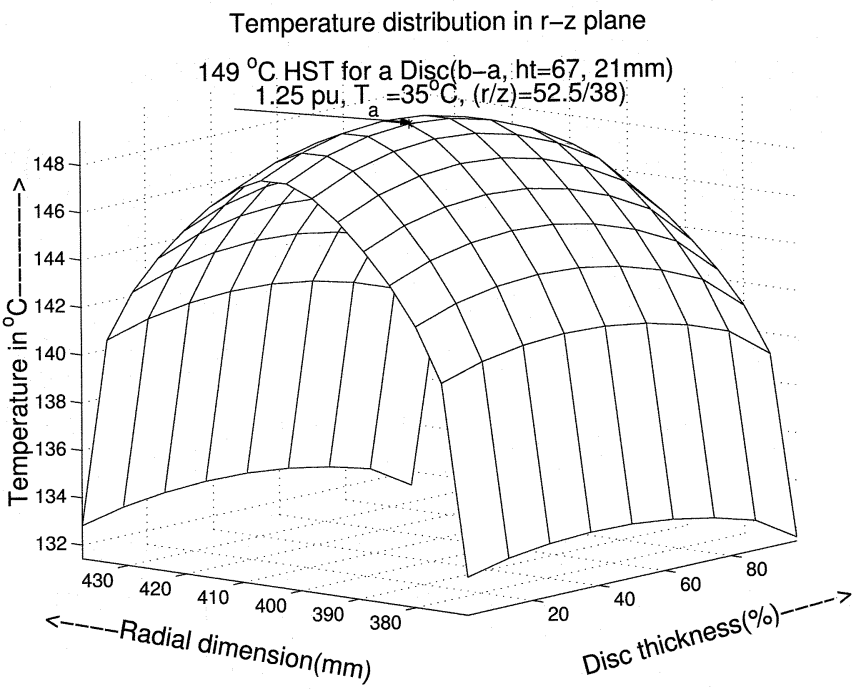

Fig. 6. Temperature distribution of a HV disc.

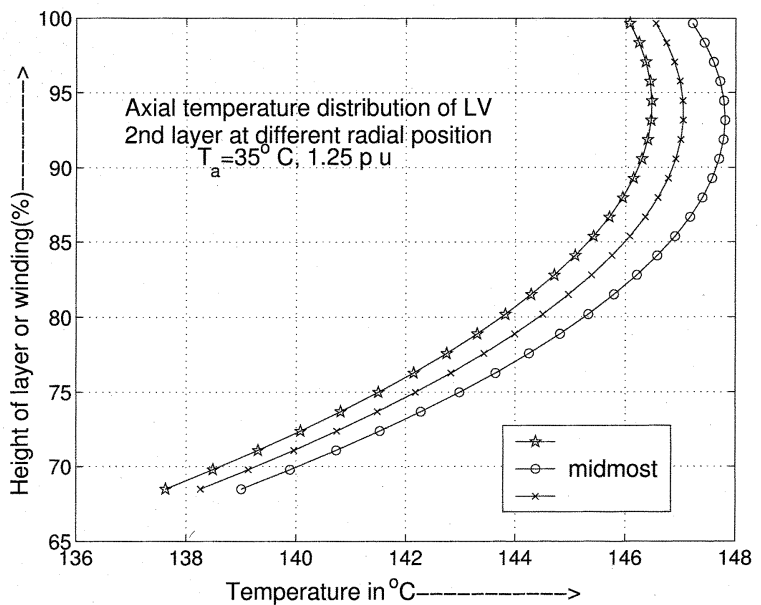

Fig. 7. Axial temperature distribution of the second layer of LV winding

temperature depends on the number of layers per disc. The radial temperature curve for different numbers of layers per disc is shown in Fig. 4. The axial temperature distribution from top to bottom of a disc in ONAF cooling mode is shown in Fig. 5. It 


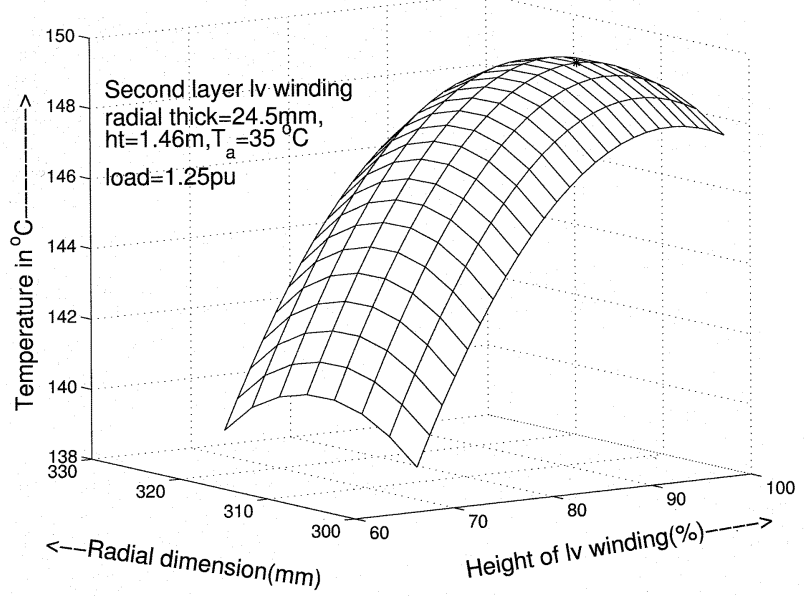

Fig. 8. Temperature distribution of LV winding layer.

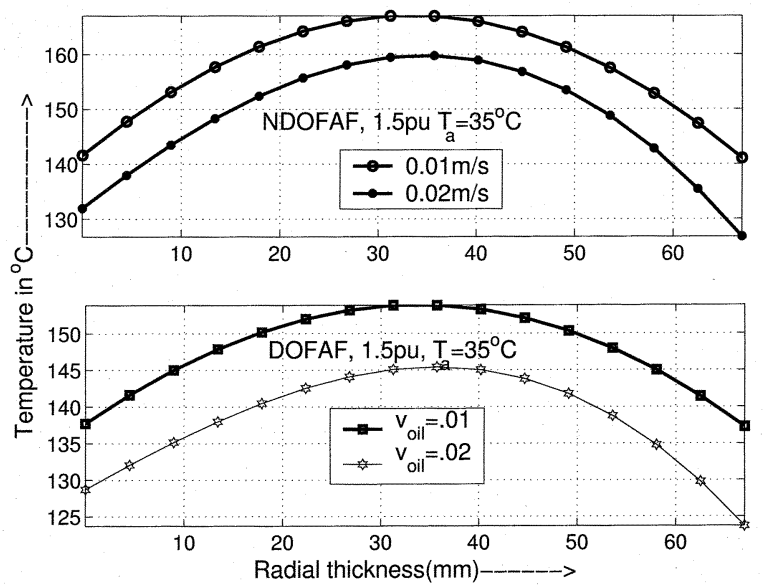

Fig. 9. Winding with 20 layers/disc, OF.

may be observed that the maximum temperature occurs at about $35-45 \%$ of thickness of the disc since the natural convection is better from the top of the disc. Fig. 6 shows the spatial temperature distribution of a disc in HV winding, which happens to be around second or third from the top which, in part, is due to absence of intense heat source above the disc. But this position would change if there is oil stagnancy in the cooling ducts. For layer winding, the axial temperature distribution is shown in Fig. 7. It can be pointed out that for layer winding, HST location is around 90 to $95 \%$ of winding from the bottom. The spatial temperature distribution for a layer winding in $\mathrm{r}$, $\mathrm{z}$ plane has been shown in Fig. 8 .

1) OF Cooling Mode: In the present work, the effect of forced cooling on the magnitude and location of HST is considered in some detail. This is a very important practical aspect and work in this area appears to be rather scanty. The results of this study is shown in Figs. 9 and 10. More complex cooling modes involving DOFAF or NDOFAF are considered as they have practical overtones. In this work, different rates of forced oil circulation are taken into account. The estimated temperature of the hot spot and its location, per se seems to be in line with the field measurements. However, there seem to be no published literature giving this information against which comparisons can be made. It is clear from Figs. 9 and

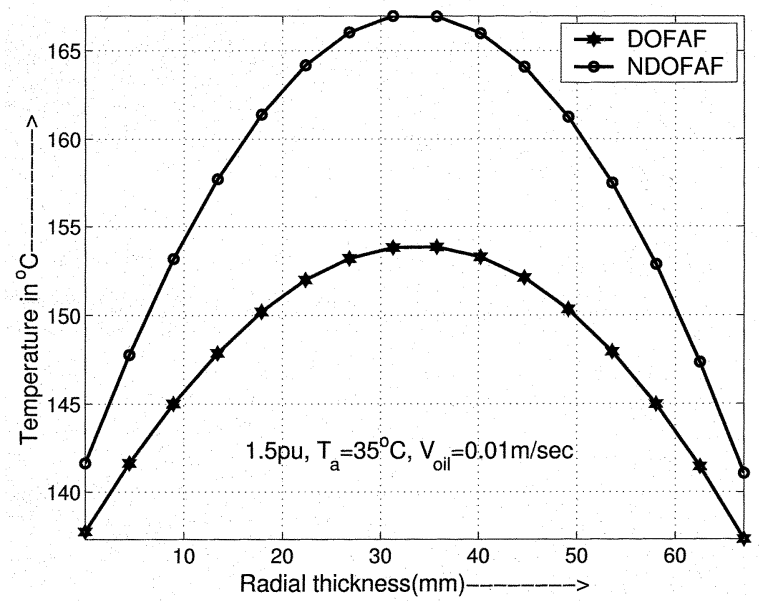

Fig. 10. Comparision of NDOFAF and DOFAF.

TABLE II

CALCULATED HST IN TRANSFORMERS Windings, ONAF

\begin{tabular}{c|c|c|c|c|c|c}
\hline $\begin{array}{c}\text { Tra } \\
\text { MVA }\end{array}$ & $\begin{array}{c}\text { load } \\
\text { p.u. }\end{array}$ & $\begin{array}{c}\text { cool- } \\
\text { ing }\end{array}$ & $\begin{array}{c}T_{a} \\
{ }^{\circ} C\end{array}$ & $\begin{array}{c}\text { wdg } \\
\text { type }\end{array}$ & $\begin{array}{c}\text { lo } c^{n} r / z \\
(\% / \%)\end{array}$ & $\begin{array}{c}\text { HST, }{ }^{o} \mathrm{C} \\
\text { propo. }\end{array}$ \\
\hline $25 / 32$ & 1 & ON & 25 & hvD & $52 / 38$ & 98 \\
$25 / 32$ & 1 & ON & 25 & lv1L & $51 / 88$ & 100 \\
$25 / 32$ & 1 & ON & 25 & lv2L & $54 / 89$ & 100 \\
$25 / 32$ & 1.25 & ON & 35 & hvD & $53 / 38$ & 149 \\
$25 / 32$ & 1.25 & ON & 35 & lv1L & $50 / 92$ & 148 \\
$25 / 32$ & 1.25 & ON & 35 & lv2L & $50 / 93$ & 149 \\
$25 / 32$ & 1.4 & ON & 35 & hvD & $50 / 40$ & 168 \\
$25 / 32$ & 1.4 & ON & 35 & lv1L & $49 / 91$ & 181 \\
$25 / 32$ & 1.4 & ON & 35 & lv2L & $49 / 91$ & 179 \\
$25 / 32$ & 1.5 & ON & 35 & hvD & $50 / 40$ & 194 \\
$25 / 32$ & 1.5 & ON & 35 & lv1L & $52 / 90$ & 206 \\
$25 / 32$ & 1.65 & ON & 35 & hvD & $52 / 42$ & 230 \\
$25 / 32$ & 1.65 & ON & 35 & lv1L & $49 / 92$ & 260 \\
\hline 22.5 & 0.8 & ON & 35 & hvD & $50 / 38$ & 75 \\
22.5 & 0.8 & ON & 35 & lvD & $50 / 40$ & 72 \\
22.5 & 1 & ON & 35 & hvD & $52 / 40$ & 100 \\
22.5 & 1 & ON & 35 & lvD & $52 / 40$ & 98 \\
22.5 & 1.25 & ON & 35 & hvD & $52 / 40$ & 112 \\
22.5 & 1.25 & ON & 35 & lvD & $52 / 40$ & 114 \\
22.5 & 1.3 & ON & 35 & hvD & $52 / 42$ & 122 \\
22.5 & 1.3 & ON & 35 & lvD & $52 / 42$ & 122 \\
22.5 & 1.5 & ON & 35 & hvD & $52 / 45$ & 141 \\
22.5 & 1.5 & ON & 35 & lvD & $52 / 42$ & 150 \\
22.5 & 1.8 & ON & 35 & hvD & $52 / 45$ & 203 \\
22.5 & 1.8 & ON & 35 & lvD & $52 / 45$ & 220 \\
22.5 & 2 & ON & 35 & lvD & $52 / 45$ & 243 \\
22.5 & 2.2 & ON & 35 & hvD & $50 / 45$ & 263 \\
22.5 & 2.2 & ON & 35 & lvD & $50 / 45$ & 292 \\
\hline & & & & & \\
\hline
\end{tabular}

10 , that the HST in the case of NDOFAF mode is greater than that of corresponding DOFAF mode, so is the average temperature rise. From this figure, it is also borne out that by an increase in the amount of cooling by way of increased oil flow speed would provide a reduced winding temperature, which is obvious. However, an increase oil flow rate has a lesser effect on HST in case of NDOFAF mode than the DOFAF mode. From the results of this investigation, it is clear that DOF cooling is more effective than NDOF cooling mode, as expected. The corresponding magnitude of HST at different loading and cooling condition is given in Table III. 
TABLE III

CAlculated HST in a HV DisC AT DifFERENT OF LEVEL

\begin{tabular}{c|c|c|c|c|c|c}
\hline $\begin{array}{c}\text { Tra } \\
\text { MVA }\end{array}$ & $\begin{array}{c}\text { load } \\
\text { p.u. }\end{array}$ & $\begin{array}{c}\text { cool- } \\
\text { ing }\end{array}$ & $\begin{array}{c}V_{\text {oil }} \\
\frac{m}{S}\end{array}$ & $\begin{array}{c}T_{a} \\
{ }^{o} C\end{array}$ & $\begin{array}{c}\operatorname{loc} c^{n} r / z \\
(\% / \%)\end{array}$ & $\begin{array}{c}\mathrm{HST}\left({ }^{\circ} \mathrm{C}\right) \\
\text { Propo. }\end{array}$ \\
\hline \hline $25 / 32$ & 1.5 & ND & .01 & 35 & $50 / 40$ & 167 \\
$25 / 32$ & 1.5 & ND & .02 & 35 & $50 / 40$ & 159 \\
$25 / 32$ & 1.65 & ND & .01 & 35 & $50 / 40$ & 210 \\
$25 / 32$ & 1.5 & DF & .01 & 35 & $50 / 50$ & 155 \\
$25 / 32$ & 1.5 & DF & .02 & 35 & $50 / 50$ & 145 \\
$25 / 32$ & 1.65 & DF & .01 & 35 & $50 / 50$ & 194 \\
22.5 & 1.6 & ND & .005 & 35 & $50 / 45$ & 143 \\
22.5 & 1.6 & ND & .02 & 35 & $50 / 45$ & 130 \\
22.5 & 1.6 & DF & .005 & 35 & $50 / 50$ & 136 \\
22.5 & 1.6 & DF & .02 & 35 & $50 / 50$ & 118 \\
\hline
\end{tabular}

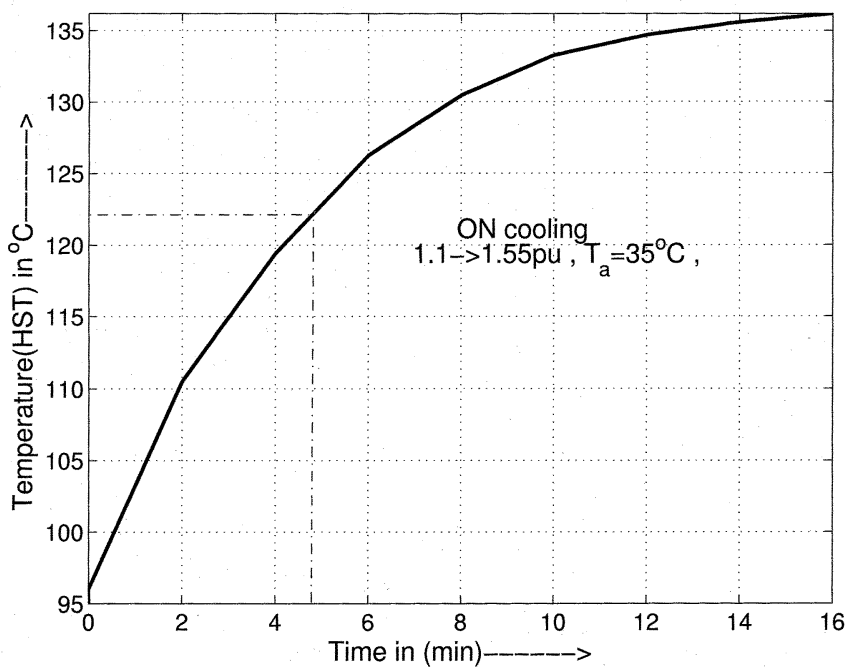

Fig. 11. Transient thermal performance of HV disc winding, ONAF.

\section{B. Unsteady State Thermal Performance}

The thermal performance in dynamic loading and dynamic ambient is of great interest. It has been assumed here that the boundary surface temperature as well as oil temperature gradient depends on time, exactly similar to the case presented by (17). Also, the method of calculation of the thermal parameters is exactly the same as that in the steady state case. In this work, heat flow in disc and layer winding has been studied extensively. When a transformer is loaded, its winding heats up. The heating process was simulated by introducing a transient thermal load. Fig. 11 shows heating curve, as a function of time. The calculations have been made under the assumption that the winding cooling duct temperature is always higher than the tank oil temperature.

\section{Comparison With Loading Guides}

Figs. 12 and 13, indicate the extent of departure of the calculated temperature from the Author's results and those obtained using the formulation available in IEEE and IEC standards, for two different transformers used in the Authors' calculation. It is to be noted that the Author's model, which takes into account many of the facts arisen here, deemed to be more accurate in comparison to those computed using the available guides and standards. Admittedly however, the comparisons shown here

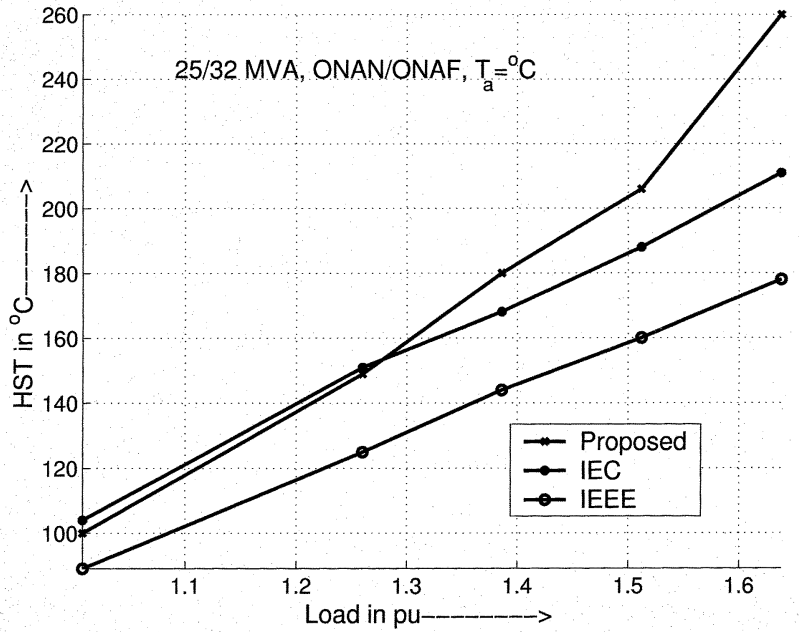

Fig. 12. HST versus load, case (I).

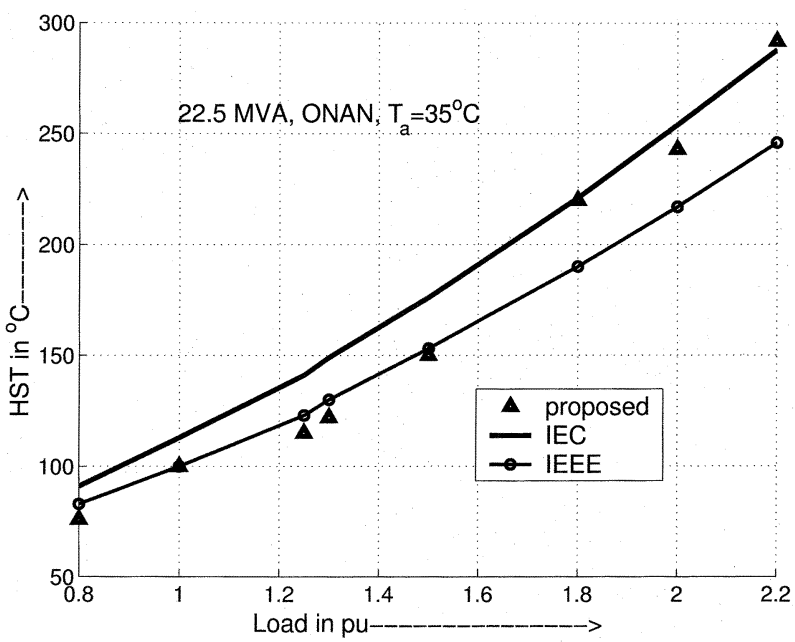

Fig. 13. HST versus load, case (II).

would be more meaningful only when experimental finding of HST can be compared with that of the proposed model and loading guides.

1) Predicting Transformer Life: After prediction of HST, the transformer remaining life can be predicted using empirical formulae. An aging acceleration factor (AAF) for insulation rated for $65^{\circ} \mathrm{C}$ average rise and $80^{\circ} \mathrm{C}$ hottest rise is given by the following equation due to Mc Nutt:

$$
A A F=\operatorname{Exp}\left\{\frac{15000}{383}-\frac{15000}{T_{h s}+273}\right\} .
$$

This factor will be nearly unity at temperature of about $110^{\circ} \mathrm{C}$. Above $110^{\circ} C$, the factor will be greater than 1.0, indicating an accelerated thermal degradation. Once the acceleration factor is known, the amount of aging can be calculated easily by multiplying this factor with period of time over which the transformer insulation was at HST. With variable loads w.r.t time, the equivalent factor can be calculated as under

$$
A A F_{\text {eqv }}=\frac{\sum_{i}^{N} A A F_{i} \triangle t_{i}}{\sum_{i}^{N} \triangle t_{i}}
$$




\section{CONCLUSION}

1) In this paper, an attempt has been made to suggest a method to improve the accuracy of prediction of the temperature of the hottest spot in power transformer by solving the BVP of heat conduction analytically.

2) The purely theoretical approach for evaluating HST and TOT, followed in this paper seems to correspond reasonably well with results of actual tests and on site measurements [5].

3) The thermal model presented here can predict the hot spot location, with a reasonable degree of accuracy and in a newly commissioned transformer, the prediction accuracy is even better.

4) The results of the present study indicate that besides assessment of thermal performance, there are distinct possibilities for planning, overload schedules on station and power transformer. Also, the authors are currently working on a technique for online estimation of temperature at different point of transformer knowing the load conditions and developing an expert system for transformer diagnosis based on HST.

\section{APPENDIX A}

The finite Hankel transform and inverse transform pair are of the form

$$
\begin{aligned}
\bar{\psi}\left(\beta_{n}\right) & =\int_{a}^{b} r \psi(r) \mathbf{K}_{0}\left(\beta_{n}, r\right) \mathbf{d} r ; \quad r \rightarrow \beta_{n} . \\
\psi(r) & =\sum_{n=1}^{\infty} \bar{\psi}\left(\beta_{n}\right) \mathbf{K}_{0}\left(\beta_{n}, r\right) ; \quad \beta_{n} \rightarrow r .
\end{aligned}
$$

Finite Fourier transform and inverse transform pair are given in (26) and (27).

$$
\begin{aligned}
\bar{\psi}\left(\alpha_{m}\right) & =\int_{0}^{\ell} \psi(z) \mathbf{M}\left(\alpha_{m}, z\right) \mathbf{d} z ; \quad z \rightarrow \alpha_{m} . \\
\psi(z) & =\sum_{m=1}^{\infty} \bar{\psi}\left(\alpha_{m}\right) \mathbf{M}\left(\alpha_{m}, z\right) ; \quad \alpha_{m} \rightarrow z
\end{aligned}
$$

where, the kernel function $\mathbf{K}_{0}\left(\boldsymbol{\beta}_{n}, r\right)$, also known as normalized Eigen function for $\mathrm{r}$ variable, is the ratio of Eigen function $\mathbf{R}_{0}\left(\boldsymbol{\beta}_{n}, r\right)$ to the square root of orthonormal $\mathbf{N}$. Mathematically

$$
\begin{aligned}
\mathbf{K}_{0}\left(\beta_{n}, r\right)= & \frac{\mathbf{R}_{0}\left(\beta_{n}, r\right)}{\sqrt{\mathbf{N}}} \\
\mathbf{R}_{0}\left(\beta_{n}, r\right)= & \frac{J_{0}\left(\beta_{n} r\right)}{h_{2} J_{0}\left(\beta_{n} b\right)-k_{2} \beta_{n} J_{1}\left(\beta_{n} b\right)} \\
& -\frac{Y_{0}\left(\beta_{n} r\right)}{h_{2} Y_{0}\left(\beta_{n} b\right)-k_{2} \beta_{n} Y_{1}\left(\beta_{n} b\right)} \\
\mathbf{N}= & \frac{b^{2}}{2}\left[\frac{h_{2}^{2}}{\beta_{n}^{2} k_{2}^{2}}+1\right] R_{0}^{2}\left(\beta_{n}, b\right) \\
& -\frac{a^{2}}{2}\left[\frac{h_{1}^{2}}{\beta_{n}^{2} k_{1}^{2}}+1\right] R_{0}^{2}\left(\beta_{n}, a\right) .
\end{aligned}
$$

And the corresponding quantities for $\mathrm{z}$ variable are as follows:

$$
\mathbf{M}\left(\alpha_{m}, z\right)=\frac{\mathbf{Z}\left(\alpha_{m}, z\right)}{\sqrt{\mathbf{N}^{\prime}}}=\frac{\cos \alpha_{m} z+\frac{h_{3} \sin \alpha_{m} z}{k_{3} \alpha_{m}}}{\sqrt{\mathbf{N}^{\prime}}}
$$

$$
\mathbf{N}^{\prime}=\frac{1}{2}\left[\left(\frac{\alpha_{m}^{2}+\frac{h_{3}^{2}}{k_{3}^{2}}}{\alpha_{m}^{2}}\right)\left(l+\frac{\frac{h_{4}}{k_{4}}}{\alpha_{m}^{2}+\frac{h_{4}^{2}}{k_{4}^{2}}}\right)+\frac{\frac{h_{3}}{k_{3}}}{\alpha_{m}^{2}}\right] .
$$

The parameter $\beta_{n}$ and $\alpha_{m}$ are the positive roots of transcendental (13) and (14), respectively, which are known as Eigenvalues. However, combine integral transforms and its inverse transform pair can be defined, (30) and (31). The detailed mathematical treatment of Eigen functions and Eigenvalues can be found in [7].

$$
\begin{aligned}
\overline{\overline{\mathbf{f}}}\left(\beta_{n}, \alpha_{m}\right) & =\int_{0}^{\ell} \int_{a}^{b} r \mathbf{f}(r, z) \mathbf{K}_{0}\left(\beta_{n}, r\right) \mathbf{M}\left(\alpha_{m}, z\right) \mathbf{d} r \mathbf{d} z \\
\mathbf{f}(r, z) & =\sum_{n=1}^{\infty} \sum_{m=1}^{\infty} \overline{\overline{\mathbf{f}}}\left(\beta_{n}, \alpha_{m}\right) \mathbf{K}_{0}\left(\beta_{n}, r\right) \mathbf{M}\left(\alpha_{m}, z\right)
\end{aligned}
$$

\section{APPENDIX B}

Mean Nusselt number of top surface of annular cylindrical winding for laminar and turbulent regime, will normally be of the form

$$
\begin{aligned}
& N u_{m}=0.54 \mathbf{R} a_{\delta}^{0.25}=0.61 \mathbf{R} a_{* \delta}^{0.2} ; \quad \delta=\frac{b-a}{2} \\
& N u_{m}=0.15 \mathbf{R} a_{\delta}^{\frac{1}{3}}=0.24 \mathbf{R} a_{* \boldsymbol{\delta}}^{\frac{1}{4}}
\end{aligned}
$$

where $\mathrm{a}$ and $\mathrm{b}$ are the inner and outer radius of annular disc or layer. Nusselt number of bottom surface of annular cylindrical winding for laminar and turbulent regime in (34)

$$
N u_{m}=0.27 \mathbf{R} a_{\delta}^{0.25}=0.35 \mathbf{R} a_{* \delta}^{0.2} ; \quad \delta=\frac{b-a}{2} .
$$

The axial oil temperature gradient in presence of cooling by a constant heat flux can be found out by using (35), due to Kimura and Bejan [9] thus

$$
\frac{\partial \mathbf{T}}{\partial z}=4.25 \times 10^{-2} \frac{q_{w}}{k_{o i l}}\left(\frac{\ell}{\pi D_{m}}\right)^{\frac{4}{9}} \mathbf{R} a_{* \ell}^{-\frac{1}{9}}
$$

where $\ell$ is the winding height, $D_{m}$ is the mean diameter of annular disc or layer of windings. Determination of boundary conditions in OF mode too require to calculate htc. While considering OF mode of cooling, it should be stressed that OF is not forced convection, in thermal engineering literature and unless oil velocity is very high. In such a case, process of cooling is even more complex. The empirical expression when the oil velocity is lower values, the mean Nusselt number based on temperature difference is of the form of (36), corresponding mean Nusselt number based on constant heat flux is of the form of (37)

$$
\begin{aligned}
N u_{m}= & 1.75\left[\mathbf{G}_{z}+0.012\left(\mathbf{G}_{z} \mathbf{G} r_{D}^{\frac{1}{3}}\right)^{\frac{4}{3}}\right]^{\frac{1}{3}} \\
& \times\left(\frac{\mu_{b}}{\mu_{w}}\right)^{0.14} \\
N u_{m}= & 1.63\left[1.8 \mathbf{G}_{z}^{.9}+.02\left(\mathbf{G}_{z} \mathbf{G} r_{* D}^{\frac{1}{3}}\right)^{1.16}\right]^{\frac{1}{3}} \\
& \times\left(\frac{\mu_{b}}{\mu_{w}}\right)^{0.125}
\end{aligned}
$$

where $\mathbf{G}_{z}=\mathbf{R} e \mathbf{P}_{r}(D / \ell)$ called Graetz number. The terms $\mu_{b}, \mu_{w}$ are viscosity of oil computed at oil bulk mean temper- 
ature of oil and at winding wall temperature, respectively. $\mathbf{R} e$ is the Reynolds number and $P_{r}$ is the prandtl number. The relative importance of natural and forced cooling is indicated by the factor $f_{r}=\mathbf{G} r_{D} / \mathbf{R} e^{2}$. If $f_{r} \approx 1$, then both of the cooling modes have to be considered. At a lower value of this factor, natural cooling can be ignored.

The oil viscosity is an important property which depends on temperature. The empirical formula to take the oil temperature variation into consideration is given in (38) below from [5]

$$
\mu=1.3573 \times 10^{-3} \operatorname{Exp}\left[\frac{2797.3}{T_{m}+273}\right] ; \quad \mu(c p), T_{m}\left({ }^{\circ} C\right) .
$$

The viscosity was calculated at the mean oil and wall temperature. Initially, the winding surface temperature is not known, so a starting guess for winding surface temperature has to be made, after calculating the value of $h$, the temperature difference $T_{w s}-T_{o i l}$ is to agree with the assumed value. The procedure has been automated using a Matlab program.

To calculate winding wall temperature at different surfaces, only bottom oil temperature is necessary. In this paper, the bottom oil rise over ambient temperature has been calculated as

$$
\Delta \theta_{b a}=\Delta \theta_{b a, r}\left[\frac{L^{2} R+1}{R+1}\right]^{\gamma} .
$$

The bottom oil exponent $\gamma$ has been assumed the same as top oil exponent. The constant $\Delta \theta_{b a, r}$, can be obtained from manufacturer's data on factory heat run tests. For the computation shown in this paper, the authors have taken the value provided in loading guides assumming it is nearly same for said $\mathrm{m} / \mathrm{c}$.

The correction factor to resistance change due to temperature at $\mathrm{H} \mathrm{S}$ location can been given, as found in [5], and is of the form of $f_{h s t}=\left(\left(p+T_{h s}\right) /\left(p+T_{h s, r}\right)\right)$.

\section{APPENDIX C}

Thermal conductivity has been treated as a vector quantity, having components in both $\mathbf{r}$ and $\mathbf{z}$ direction. Resultant thermal conductivity of the system can be estimated as $\mathbf{K}=\sqrt{k_{r}^{2}+k_{z}^{2}}$, where

$$
\begin{aligned}
k_{z} & =\frac{k_{c u} k_{i n}\left(t_{c u}+t_{i n}\right)}{t_{i n} k_{c u}+t_{c u} k_{i n}} ; \\
k_{r} & =\frac{\log \frac{r_{n}}{r_{1}}}{\left(\frac{\log \frac{r_{2}}{r_{1}}}{k_{1}}+\frac{\log \frac{r_{3}}{r_{2}}}{k_{2}}+\ldots+\frac{\log \frac{r_{n}}{r_{n}-1}}{k_{n}}\right)} .
\end{aligned}
$$

The calculation of diffusivity of insulation-conductor system involves calculation of its thermal conductivity, density, and specific heat. The equivalent mass density of the system can be cal- culated as the total mass of disc or layer divided by volume of disc or layer. The specific heat can be calculated as a weighted average value $C_{p e q}=\left(C_{p c u} M_{c u}+C_{p i n} M_{i n}\right) /\left(M_{c u}+M_{i n}\right)$.

\section{REFERENCES}

[1] IEEE Loading Guide for Mineral Oil Immersed Transformer, C57.91, pp. 18-19, 46-53, 1995.

[2] B. C. Lesieutre, W. H. Hagman, and J. L. Kirtley, "An improved transformer TOT modeling for use in an on line monitoring and diagnostic system," IEEE Trans. Power Delivery, vol. 12, pp. 249-256, Jan. 1997.

[3] D. J. Tylavsky, Q. He, J. Si, G. A. McCulla, and J. R. Hunnt, "Transformer top-oil temperature modeling and simulation," IEEE Trans. Ind. Applicat., vol. 36, pp. 1219-1225, Sept./Oct. 2000.

[4] Q. He, J. Si, and D. J. Tylavsky, "Prediction TOT for transformers using neural networks," IEEE Trans. Power Delivery, vol. 15, pp. 1205-1211, Oct. 2000.

[5] L. W. Pierce, "An investigation of the thermal performance of an oil filled transformer winding," IEEE Trans. Power Delivery, vol. 7, pp. 1347-1358, July 1992.

[6] M. V. Thaden, S. P. Meheta, S. C. Tuli, and R. L. Grubb, "Temperature rise test on a OFAF core-form transformer, including loading beyond name plate," IEEE Trans. Power Delivery, vol. 10, pp. 913-919, Apr. 1995.

[7] M. K. Pradhan, "Condition Monitoring of Power and Generator Transformers Based on the Temperature of the Hottest Spot (HST)," M.Sc. Eng. Thesis, Department of High Voltage Engineering, Indian Institute of Science, Bangalore, India, Feb. 2002.

[8] Y. A. Mikhailovsky, A. B. Shvidler, G. E. Tarle, V. M. Tchornogotsky, I. D. Voevodin, and I. S. Lyubline, "Methods of temperature rise calculation of power transformer winding," in Proc. 30th Session, vol. 1, sec. $12-16,1984$, pp. $1-6$

[9] A. Bejan, Convective Heat Transfer. New York: Wiley, 1995, pp. 177-203, 235-259.

[10] M. N. Ozisik, Heat Transfer-A Basic Approach. New York: McGrawHill, 1985, pp. 416-462.

M. K. Pradhan was born in Balasore, Orissa. He received the B.E. degree in electrical engineering from the University College of Engineering (UCE) Burla, Orissa, in 2000, and the M.Sc. degree in engineering from the High Voltage Engineering Department at the Indian Institute of Science, Bangalore, in 2002. $\mathrm{He}$ is currently pursuing the Ph.D. degree at the Indian Institute of Science, Bangalore.

He worked on the project "Building cognition of mobile Robert in a priori known environment," in UCE Burla, from 1999 to 2000. His research interests include diagnostic testing and condition monitoring (DTCM) of power equipment, artificial intelligence (AI) techniques applied to DTCM, and stochastic modeling.

T. S. Ramu was born in Bangalore, India. He received the B.E. degree from Mysore University, India, the M.E. degree from the Indian Institute of Science, Bangalore, India, and the Ph.D. degree from the Indian Institute of Technology, Madras, India.

Currently, he is a Professor in the Department of High Voltage Engineering at the Indian Institute of Science. 